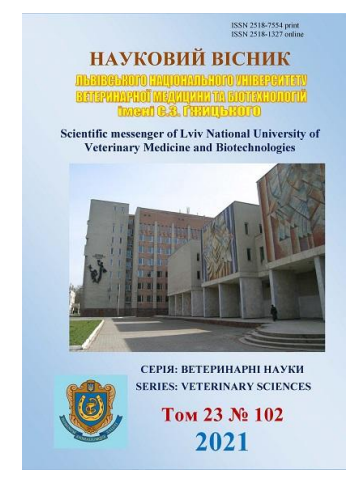

\section{Науковий вісник Аьвівського національного університету ветеринарної медицини та біотехнологій імені С.3. Гжицького. Серія: Ветеринарні науки}

\author{
Scientific Messenger of Lviv National University \\ of Veterinary Medicine and Biotechnologies. \\ Series: Veterinary sciences
}

doi: 10.32718 /nvlvet10220 https://nvlvet.com.ua/index.php/ournal

\title{
Design of Hapten Synthesis and Antibody Characterization of G-group Aflatoxins
}

\author{
Yanan Wang \\ Faculty of Veterinary Medicine, Sumy National Agrarian University, Sumy, kraine \\ College of Animal Science and Veterinary Medicine, Henan Institute of Science and Technology, Xinxiang, China
}

Article info

Received 13.05.2021

Received in revised form 17.06.2021

Accepted 18.06.2021

Faculty of Veterinary Medicine Sumy National Agrarian University, Herasima Kondratieva Str., 160, Sumy, 40021, Ukraine.

College of Animal Science and Veterinary Medicine, Henan Institute of Science and

Technology, Xinxiang. 453003, China.

Tel.: +38-099-238-62-24 E-mail:wyn564@126.com
Wang, Y. (2021). Design of Hapten Synthesis and Antibody Characterization of G-group Aflatoxins. Scientific Messenger of Lviv National University of Veterinary Medicine and Biotechnologies. Series: Veterinary sciences, 23(102), 130-135. doi: 10.32718/nvlvet10220

Food and feed contamination with Aflatoxins pose a serious threat to human health and animal husbandry development and has caused widespread concern, among them, G-group Aflatoxins as the main pollutant has attracted more and more attention. In order to establish a rapid, sensitive, specific and efficient immunoassay method for G-group aflatoxins, this study aimed to designed to synthesize 3 immunogens and coating antigens and identified by UV and SDS-PAGE. Then used to immunize Balb/c mice with prepared of three immunogen the titers were determined by indirect ELISA and the sensitivity was determined by competitive indirect ELISA (icELISA), the specificity was assessed by the cross-reaction test $(C R)$. The results of UV and SDS-PAGE showed that the three immunogens and the corresponding coated antigens were successfully synthesized and the best one was SA method among three synthesis methods of G-group AF artificial antigen and its conjugation ratio of AFG1 to BSA was about 5.64:1. The immune efficacy of SA method was the best, its AFG1 pAb had high titers of $1:\left(6.4 \times 10^{3}\right)$ by indirect ELISA, a good sensitivity with the $50 \%$ inhibition concentration(IC50) of $13.6 \mu \mathrm{g} / \mathrm{kg}^{-1}$ to AFG1 by blocking ELISA and a high CR to AFG2 of $82.19 \%$, it showed high specificity for other aflatoxins. The experimental results not only obtained the ideal $G$ group aflatoxin antibody, but also established a substance and technology foundation for $G$ group aflatoxin immunization methods, and can be referenced in the similar tests.

Key words: G-group Aflatoxins, Hapten, Artificial Antigen, Polyclonal Antibody, Specificity Analysis.

\section{Introduction}

Aflatoxins (AFs) has acute, chronic, carcinogenic and immunosuppressive toxic effects on human health. Among them, AFB1 has the strongest toxicity and wide pollution, accounting for more than $50 \%$. Most countries have made clear provisions on the maximum residue limit (MRL) of AFB1 in food (Wu et al., 2013; Rushing \& Selim, 2019). AFs produced under natural conditions mainly includes AFB1, AFB2, AFG1 and AFG2. The characteristics of contaminated food are that a variety of toxins almost exist at the same time and have toxic additive effects. It has become a development trend to formulate MRL and corresponding detection methods for total aflatoxins (TAFs) (AFB1 + AFB2 + AFG1 +AFG2) (Aiko \& Mehta, 2015; Xie et al., 2016). To 2013, 91 countries have adopted the TAFs limit standard, such as the Codex Alimentarius Commission (CAC) and the U.S. Food and Drug Administration (FDA), stipulated that the TAF MRL for all foods should not exceed $15 \mu \mathrm{g} / \mathrm{kg}$ (FDA,
2018), Japan's food TAF MRL should not exceed $10 \mu \mathrm{g} / \mathrm{kg}$ (Japan, 2011), the TAF MRL set by European Committee (EC) was no more $4 \mu \mathrm{g} / \mathrm{kg}$ (EC, 2006). China's existing standard has not yet involved TAFS limited requirements, but the detection method of TAF MRL in grain was prescribed in "LS/T 6128-2017: Determination of aflatoxin B1, B2, G1, and G2 in grain: ultra-high performance liquid chromatograph (China, 2017).

The analytical methods of TAFs in food mainly include physical and chemical analysis and immunoassay. in which the immune analysis method with strong selectivity, high sensitivity, fast, simple, sample screening large amount, can the advantages of the site operation, has become a hot topic, and played an important role in rapid detection of TAFs (Siahi Shadbad et al., 2012). High quality antibody is the core reagent for establishing immunoassay methods. In terms of current research progress, one of the main ways to realize TAFs immunoassay is to prepare single antibodies with high 
sensitivity and specificity for B group AF and G group AF respectively, After that, form mixed universal antibodies, but the high quality antibody is prepared from semi-antigen molecular design and antigen synthesis (Gefen et al., 2015). The synthesis methods of G AF antigen have been reported, but the research on comparative analysis of different semi-antigen synthesis, antigen synthesis and antibody characteristics has not been reported. In this study, AFG1 was reactive starting raw materials, which was designed to synthesize artificial antigen by different AFG1 semi-antigen molecular design and antigen synthesis methods, and prepared polyclonal antibody (pAb), analyze its characteristics, and select the best hapten molecular design and antigen synthesis method, In combination with antigen synthesis, the preparation of G-group AF high-quality antibodies with strong specificity, high affinity, and it laid a foundation for the establishment of TAFS immunoassay.

\section{Materials and methods}

Reagents. AFB1, AFB2, AFG1 and AFG2 standard products, products of german prio lab company, purchased from Zhengzhou Yingke reagent consumables business department; Bovine serum albumin (BSA), ovalbumin (OVA), Freund's complete adjuvant (FCA), Freund's incomplete adjuvant (FIA), products of Pierce CompanyPurchased from Yunke reagent consumables station, Zhongyuan District, Zhengzhou ; and goat antimouse IgG conjugated with horseradish peroxidase (GaMIgG-HRP) were obtained from Huamei Biological Engineering Co., Ltd. Other reagents were commercially available, and were analytical or chromatographic pure, the test water is triple distilled water.

Solutions. The diluent used in the enzyme-linked immunosorbent assay (ELISA) is $0.01 \mathrm{~mol} \cdot \mathrm{L}-1 \mathrm{pH} 7.4$ phosphate buffer solution (PBS); the washing solution is PBS containing $0.5 \mathrm{~g} / \mathrm{L}$ Tween-20 (PBST); Blocking buffer containing swine serum $(5 \%, \mathrm{v} / \mathrm{v})$ in PBST; the coating solution is $0.1 \mathrm{~mol} / \mathrm{L}$ carbonate buffer solution (CBS) with $\mathrm{pH} 9.6$.

Experimental instrument. Pharmacia protein nucleic acid analyzer, Amersham company; Wd-9403d ultraviolet instrument, Beijing Liuyi Instrument Factory; Rf-5301 PC fluorescence spectrometer, Shimadzu company, Japan; Jy-3000 electrophoresis instrument, Beijing Junyi Dongfang electrophoresis equipment Co., Ltd; F-4500 fluorescence spectrophotometer, Hitachi, Japan; Multifunctional automatic microplate reader (MK3), was purchased from Thermo in USA.

Experimental animals. SPF 4-week-old female Balb/C mice, Provided by Experimental Animal Center of Xinxiang Medical College, Animal License Number: SCXK (Yu) 2010-0002.

The haptens molecular design and artificial antigen synthesis of G-group AF. AFG1 was selected as the starting material, according to the active groups and sites on its molecular structure (see Figure 1), and on the basis of reference to relevant literature, the molecular design of AFG1 hapten and the synthetic methods of artificial immunogen were mainly semi acetal (SA) and epoxide (EP), enol ether derivative (EED) and other methods to synthesize AFG1-BSA.<smiles>COc1cc2c(c3oc(=O)c4c(=O)occc4c13)C1C=COC1O2</smiles>

Fig. 1. Molecular structure of AFG1

SA method. AFG1 is converted to AFG2a under the action of $\mathrm{H} 2 \mathrm{SO} 4$, the aldehyde group of afg2a and the amino group of BSA form unstable Schiff base. Under the reduction action of $\mathrm{NaBH} 4$, which formed a stable AFG2A-BSA (Chu et al., 1985; Li et al., 2015). The synthesis route is shown in Figure 2.

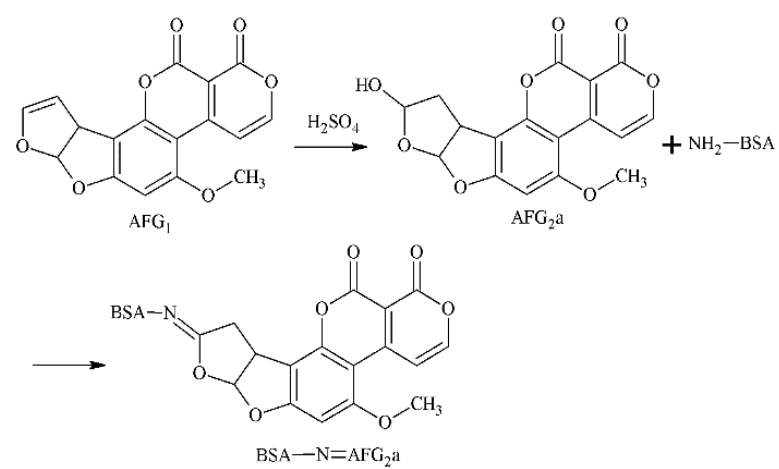

Fig. 2. The synthetic route of AFG1-BSA by SA method

EP method. Using the double furan ring double bond of AFG1, under the action of oxidation, the 3 and 4 positions of AFG1 form epoxides, react with - NH2 of BSA to form secondary amines, and couple with BSA in the form of - CONH - to synthesize AFG1-BSA. The synthesis route of (Martin \& Garner, 1977; Zhang et al., 2009) is shown in the figure 3.

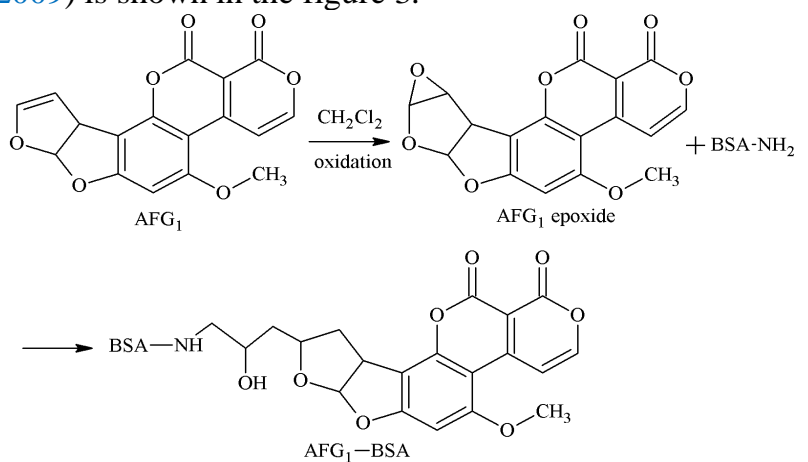

Fig. 3. The synthetic route of AFG1-BSA by EX method

EED method. The AFG1 4-bit active site is obtained by adding an ethanolic acid attachment arm to obtain an AFG1-GA, and the carboxyl group of AFG1-Ga was conjugated with BSA (Iyer \& Harris, 1993). The synthesis route of is shown in the figure 4. 


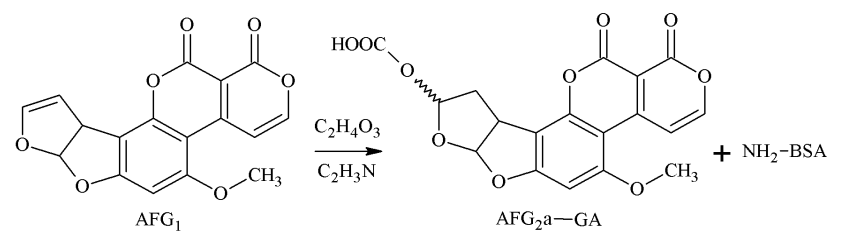<smiles>COc1cc2c(c3c1oc(=O)c1c(OC)cccc13)C1C[C@H](O[13CH3])OC1O2</smiles>

Fig. 4. The synthetic route of AFG1-BSA by EED method

Identification of G-group AF artificial antigen. UV identification. A certain amount of AFG1, BSA and AFG1-BSA, and AFG1were weighed and dissolved with methanol the methanol as a blank control; BSA and AFG1-BSA were dissolved in methanol PBS solution [V (methanol) : V (PBS) = 4:6)], which was used as blank control. Uv scanning was performed at $220 \sim 450 \mathrm{~nm}$ and analyze the scanning atlas, refer to the method of Wang Yanan et al. calculate the molecular binding ratio of AFG1 and BSA (Wang et al., 2014; 2016).

SDS-PAGE identification. The concentration of concentrated glue is $50 \mathrm{~g} / \mathrm{L}^{-1}$, voltage $90 \mathrm{~V}$; separation glue mass concentration is $120 \mathrm{~g} / \mathrm{L}^{-1}$, voltage $45 \mathrm{~V} ; 10 \mu \mathrm{L}$ per hole per hole, $10 \mu \mathrm{g}$ of protein, Coomassie blue staining, the molecular binding ratio of AFB1 and BSA was calculated by UV gel imaging system analysis software.

Fluorescence intensity identification. A certain amount of BSA and AFG1-BSA were weighed and dissolved in methanol PBS solution [v (methanol) : v $(\mathrm{PBS})=4: 6)]$, The mass concentration was $1.0 \mathrm{mg} \bullet \mathrm{mL}-$ 1 , and the fluorescence intensity was measured at the excitation wavelength of $365 \mathrm{~nm}$ and emission wavelength of $440 \mathrm{~nm}$.

Analysis of Preparation and Immunological Characteristics of AFG1 pAb. Balb/C mice were immunized with the antigen AFG1-BSA synthesized by three different methods, and each antigen was immunized with 1 group. There were 3 groups with 5 mice in each group.The immunization dose was calculated according to the amount of BSA in AFG1-BSA, $50 \mu \mathrm{g}$ for each, with a volume of $0.2 \mathrm{ml}$. Subcutaneous injection was performed at $4 \sim 6$ points on the back for 5 times, with a time interval of $4 \mathrm{w}$. The suborbital sinus of the eyeball was removed 21 days after the last immunization, and blood samples were collected, and serum was separated to obtain AFG1 pAb.

Analysis of immunological characteristics of AFB1 pAb. The titer was determined by indirect ELISA (Zhang et al., 2008) Sensitivity was determined by indirect competitive ELISA (icELISA) to determine the $50 \%$ inhibition concentration $\left(\mathrm{IC}_{50}\right)$ of AFG1 pAb to AFG1, and sensitivity was measured by $\mathrm{IC}_{50}$ ( $\mathrm{Li}$ et al., 2015). Specificity identification, AFG1, G2, B1 and B2 were selected as inhibitors by cross reaction test. The $\mathrm{IC}_{50}$ of each inhibitor was determined by icELISA. The cross- reaction rate $(\mathrm{CR} \%)$ was the percentage of $\mathrm{IC}_{50}$ of $\mathrm{AFG1}$ PAB to AFG1 and IC50 of AFG2, AFB1 and AFB2.

\section{Results}

UV identification. It can be seen from Figure 5 that the maximum absorption peak of BSA is at $278 \mathrm{~nm}$, $\mathrm{AFG}_{1}$ has four absorption peaks at 243, 257, 264 and $362 \mathrm{~nm}$ between 200 and $500 \mathrm{~nm}$, and the maximum absorption peak is at $362 \mathrm{~nm}$. AFG1-BSA (SA) and AFG1-BSA (EP) have characteristic absorption peaks at $346 \mathrm{~nm}$ and $413 \mathrm{~nm}$, and AFG1-BSA (EED) has characteristic absorption peaks at $423 \mathrm{~nm}$. The three methods all have different UV absorption characteristics from BSA and AFG1, indicating that the artificial antigen AFG1-BSA was successfully synthesized by the three methods. The calculated molecular binding ratio of AFG1 and BSA was 5.64:1 (AFG1-BSA(SA)), 7.17:1 (AFG1$\mathrm{BSA}(\mathrm{EP}))$ and 3.43:1 (AFG1-BSA(EED)).

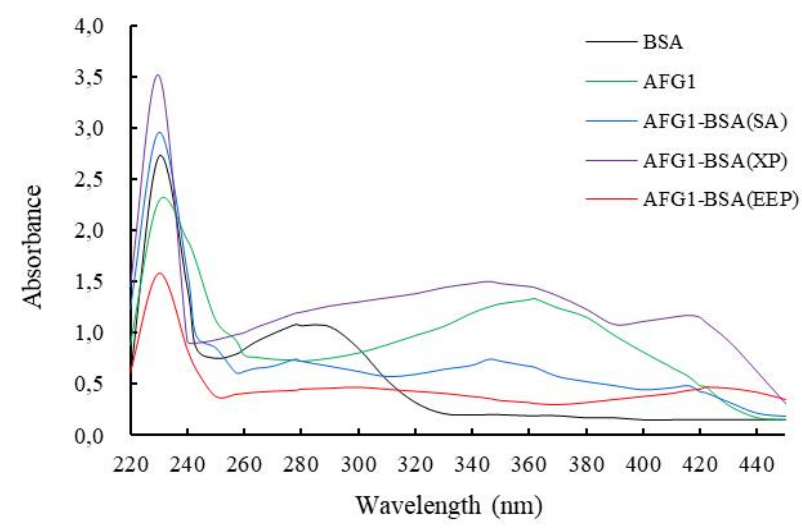

Fig. 5. UV spectra of artificial antigen AFG1-BSA

SDS-PAGE identification. It can be seen from Figure 6 that the migration rate of the immunogen AFG1-BSA synthesized by the three methods on the gel plate is less than that of BSA, indicating that the MW of AFG1-BSA is greater than that of BSA, this indicates that the synthesis of AFG1-BSA is successful.

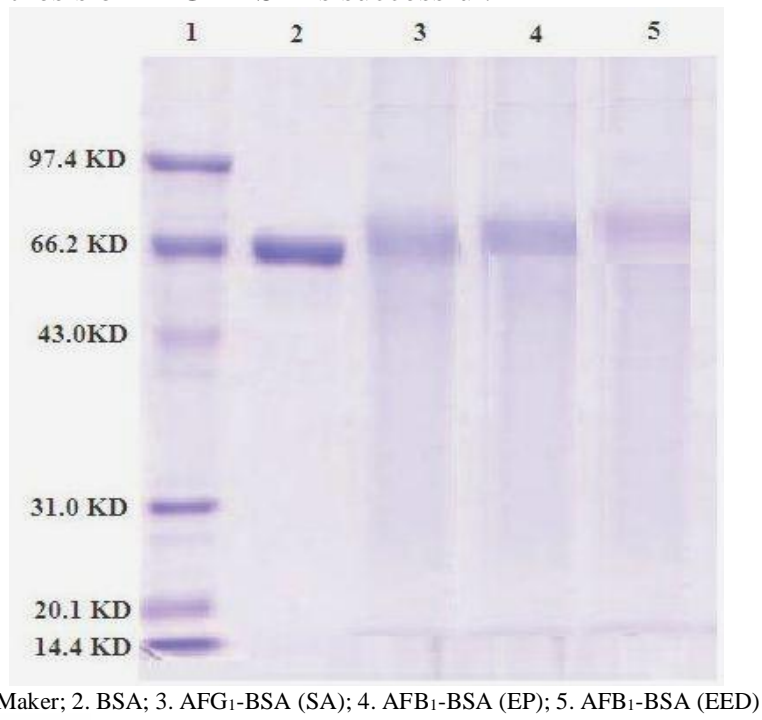

Fig. 6. The photo of artificial antigen AFG1-BSA by SDS-PAGE 
Fluorescence intensity identification. It can be seen from Figure 7 that under the same excitation light source and the same protein mass concentration $(1.0 \mathrm{mg} / \mathrm{mL})$ conditions, compared with BSA, the antigens AFG1-BSA (SA), AFG1-BSA (EP), and AFG1-BSA (EED) synthesized by the three methods have enhanced fluorescence intensity to different degrees, indicating that the complete antigen synthesis was successful.

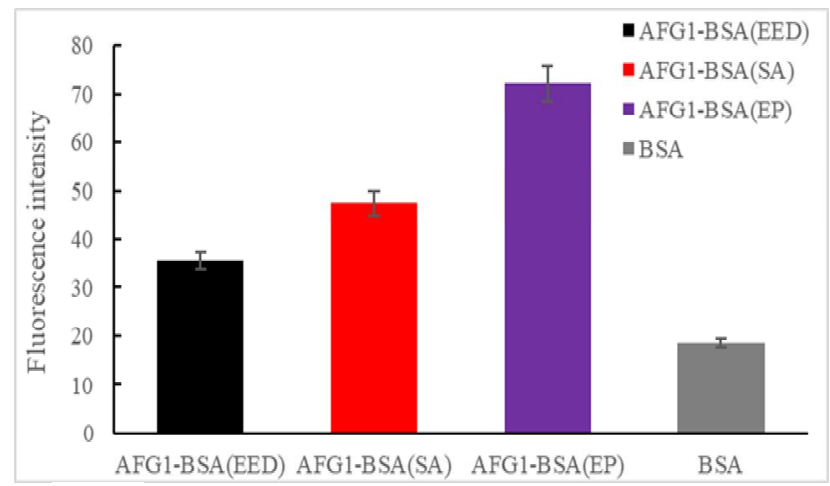

Fig. 7. The measurement of fluorescence intensity of artificial antigen AFG1-BSA

Titer determination. It can be seen from Figure 8 that after 5 immunizations, 1 mouse with the highest titer was selected from each group, and a total of 3 mice were selected. The titer was measured by indirect ELISA and compared. The AFG1 pAb titer of 3 mice all reached $1:(1.6 \times 103)$ or more, it shows that the three synthetic complete antigens AFG1-BSA all have good immunogenicity. The immune effect of the three groups is evaluated according to the titer, and the order is AFG1BSA (SA), AFG1-BSA (EP) and AFG1-BSA (EED).

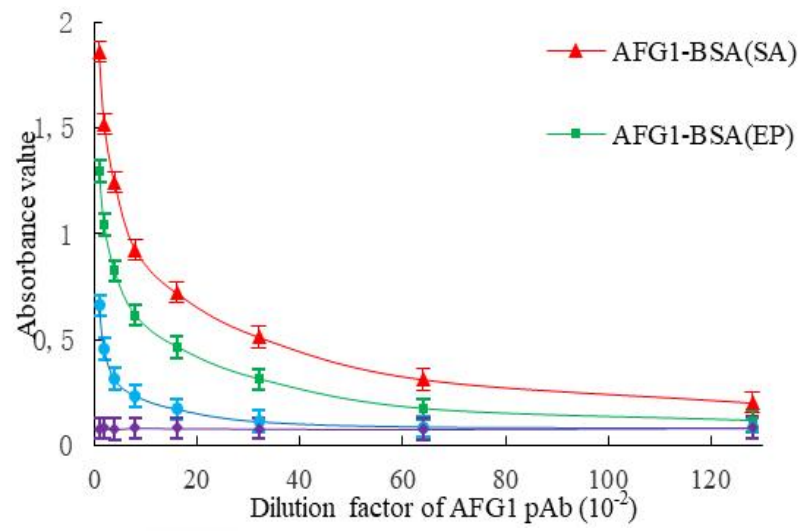

Fig. 8. The titer curves of AFG1 pAb
Sensitivity analysis. It can be seen from Figure 9 that the icELISA curve of the 3 mice with the highest titer selected after immunization showed a good linear relationship. The $\mathrm{A}_{450 \mathrm{~nm}}$ value was converted to $\mathrm{B} / \mathrm{B} 0 \%$, and then the regression analysis was performed on $\mathrm{Lg}$ [AFG1/100], all meet the criteria of linear relationship. The regression equation, $\mathrm{R}^{2}$ value and $\mathrm{IC}_{50}$ value of the three mice's serum AFG1 pAb inhibition curve are shown in Table 2. Among them, the serum of the AFG1-BSA (SA) group mouse has the best inhibition effect, with an IC50 of $17.43 \mu \mathrm{g} / \mathrm{kg}^{-1}$, Followed by the $\mathrm{IC}_{50}$ of the AFG1BSA (EP) group, which was $25.11 \mu \mathrm{g} / \mathrm{kg}^{-1}$, and the $\mathrm{AFG}_{1}$ BSA (EED) group had a poorer sensitivity, which was $70.41 \mu \mathrm{g} / \mathrm{kg}^{-1}$.

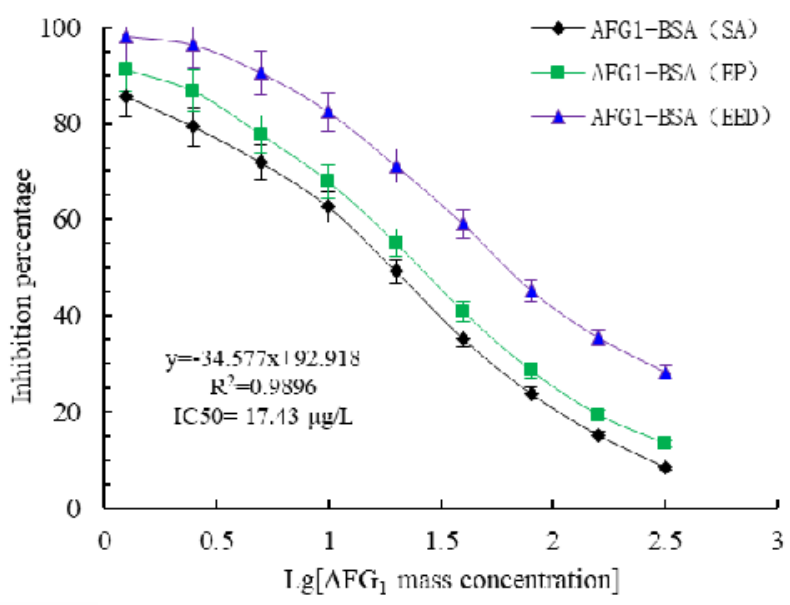

Fig. 9. The inhibition curves of AFB1 pAb against AFB1

Specificity analysis. It can be seen from Table 1 that the AFG1 pAbs obtained after the three coupling methods synthesize antigens to immunize animals can recognize AFG1 $100 \%$, and AFG1 pAb (SA) has the best sensitivity, with $\mathrm{IC}_{50}$ of $17.43 \mu \mathrm{g} / \mathrm{kg}-1$, the broad spectrum is also the best, the CR with AFG2 is $94.47 \%$, and the CR of AFB1 and AFB2 are both less than $10 \%$. AFG1 pAb (EP) has good sensitivity, with $\mathrm{IC}_{50}$ of $25.11 \mu \mathrm{g} / \mathrm{kg}-1$, but it has poor broad-spectrum, and its CR with other AFs is less than $10 \%$. $\mathrm{AFG}_{1} \mathrm{pAb}$ (EED) has poor sensitivity to $\mathrm{AFG}_{1}$, with $\mathrm{IC}_{50}$ of $70.41 \mu \mathrm{g} / \mathrm{kg}-1$. The results show that AFG1-BSA prepared by hemiacetal method has high sensitivity, strong specificity and best broad spectrum.

Table 1

The percent cross-reactivity of AFG1 pAb with AFB1, AFB2, AFG1,AFG2

\begin{tabular}{|c|c|c|c|c|c|c|}
\hline \multirow[b]{2}{*}{$\mathrm{AF}$} & \multicolumn{2}{|c|}{ AFG1 pAb（SA） } & \multicolumn{2}{|c|}{ AFG1 pAb (EP) } & \multicolumn{2}{|c|}{ AFG1 pAb (EED) } \\
\hline & $\begin{array}{c}I C_{50} / \\
\left(\mu \mathrm{g} \cdot \mathrm{kg}^{-1}\right)\end{array}$ & Cross-reactivity & $\begin{array}{c}I C_{50} / \\
\left(\mu \mathrm{g} \bullet \mathrm{kg}^{-1}\right)\end{array}$ & Cross-reactivity & $\begin{array}{c}I C_{50} / \\
\left(\mu \mathrm{g} \bullet \mathrm{kg}^{-1}\right)\end{array}$ & Cross-reactivity \\
\hline B1 & $>1 \times 10^{3}$ & $<10$ & $>1 \times 10^{3}$ & $<10$ & $>1 \times 10^{3}$ & $<10$ \\
\hline B2 & $>1 \times 10^{3}$ & $<10$ & $>1 \times 10^{3}$ & $<10$ & $>1 \times 10^{3}$ & $<10$ \\
\hline G1 & 17.43 & 100 & 25.11 & 100 & 70.41 & 100 \\
\hline G2 & 18.45 & 94.47 & $>1 \times 10^{3}$ & $<10$ & $>1 \times 10^{3}$ & $<10$ \\
\hline
\end{tabular}




\section{Discussion}

About AF artificial antigen synthesis pathway. High-quality antibodies are the core reagents for establishing immunoassay methods. In terms of current research progress, there are two ways to realize immunoassay of total TAFs. One is to prepare single antibodies with high sensitivity and strong specificity for group B AFs and group G AFs, and then mix and use mixed universal antibodies. This method is currently more commonly used and has achieved good application effects ; The second is to prepare a single universal antibody that can simultaneously recognize AFB1, AFB2, AFG1, and AFG2 with high sensitivity and broad recognition spectrum. This is the most ideal technical method, but it is difficult to prepare such high-quality monoclonal antibodies. Since AF is a small molecule hapten, it is necessary to synthesize an artificial immunogen to assist the proliferation and differentiation of $\mathrm{B}$ cells with the help of activated $T$ cells to obtain qualified antibodies.

About $G$ group AF artificial immunogen synthesis method and immune effect analysis. Due to the late start of the study of G-group AF hapten molecular design, antigen synthesis methods and the characteristics of the antibodies produced, comprehensive analysis of the above three methods, the author believes that the hemiacetal method is the most effective. AFG1 is acidified to form AFG2a, using the 3-hydroxyl active group of AFG2a to synthesize antigen with Schiff's base as the spacer arm, the produced antibody has the characteristics of high titer, good sensitivity, strong specificity and broad recognition spectrum. The epoxide method can induce the body to rapidly produce antibodies, but its sensitivity is not as good as the hemiacetal method. The enol ether derivative method has obvious defects, the ability to induce the body to produce antibodies is poor, and the antibodies produced are less sensitive to AFG1. Therefore, this method has academic value for scientific research, but it is rarely applied in actual production.

About the development trend of AF artificial immunogen synthesis method. Due to the AFB1 is closely related to other AF pollution, AFB2 pollution is accompanied by AFB1, and high concentrations of AFB1 have an inhibitory effect on AFG1 and AFG2. Therefore, there are two immunological detection and evaluation methods for AF contamination in food. Some countries, including China, adopt AFB1 limit standards. However, in order to solve the simultaneous existence of multiple toxin pollution and the toxic additive effect and the corresponding lack of detection standards, some countries adopt the total amount of AF $\left(B_{1}+B_{2}+G_{1}+G_{2}\right)$ limit standard. In view of this, the focus of the research of $\mathrm{AF}$ immunoassay technology is to improve the sensitivity and specificity of antibody recognition to AFB1 through the design of AF hapten molecules and the synthesis of antigens, and to meet the requirements of the $\mathrm{AFB}_{1}$ limit standard, in the meanwhile, improve the sensitivity and broad-spectrum recognition of antibodies to AFB1, AFB2, AFG1, and AFG2, and meet the requirements for the total amount of AF. At present, the molecular design and antigen synthesis of AF hapten still remain at the level of empirical design and predictive design, and trialand-error assays are mostly used. Although a variety of identification methods such as infrared (IR), ultraviolet (UV), mass spectrometry (MS), gel electrophoresis (SDSPAGE) and nuclear magnetic resonance (NMR) have been established for synthetic artificial antigens, but in the end, it is necessary to verify its scientific rationality through animal experiments, there will be blindness and contingency to a certain extent (Guan et al., 2013). With the development of new disciplines such as molecular immunology, quantum chemistry, and molecular mechanics, as well as the popularization and application of molecular information technology, molecular simulation technology, and computer-aided technology, these provide theories and methods for reference to improve the rationality, timeliness and predictability of hapten molecular design and antigen synthesis (Xu et al., 2009; Kortkhonjia et al., 2013; Sevy \& Meiler, 2014). Kim et al. (2011) used computer-aided technology to prepare TAFs mAbs with strong specificity and broad recognition spectrum. The $\mathrm{IC}_{50}$ for AFB1, AFB2, AFG1, and AFG2 were 4.36, 7.22, 6.61, $29.41 \mu \mathrm{g} / \mathrm{kg}^{-1}$, respectively, ELISA detection technique to achieve TAFs mode. Zhou Qian et al. (2010) successfully prepared Ggroup $\mathrm{AF} \mathrm{mAb}$ with the help of molecular simulation technology, with $\mathrm{IC}_{50}$ of 17.18 and $19.75 \mu \mathrm{g} / \mathrm{kg}^{-1}$ for AFG1 and AFG2, respectively, and established an AF Ggroup ELISA detection method.

\section{Conclusion}

This study refers to a large number of literatures, according to the molecular structure and active site of AFG1, the SA method, EP method and EED method are used to prepare the $\mathrm{G}$ group $\mathrm{AF}$ artificial immunogen AFG1-BSA. The synthetic artificial antigens were identified by UV, SDS-PAGE and fluorescence intensity measurement. The results showed that among the three methods for the synthesis of G-group AF artificial immunogens, the SA method had the best effect, and the molecular binding ratio of AFG1 to BSA was 5.64:1, the AFG1 pAb produced by animal immunization has the characteristics of high titer, sensitivity, specificity, and broad spectrum. The research results establish a basis for the AF Group G immunoassays and the total amount of AF.

\section{Author Contributions}

Yanan Wang participated, original draft preparation, writing, and software. The author has read and agreed to the published version of the manuscript.

\section{Acknowledgments}

This research is funded by the National Natural Science Foundation of China (31702263), The 13th FiveYear National Key Research and development plan food safety technology research and development major project (2019YFC1605705), and The Program for Innovative Research Team (in Science and Technology) at the University of Henan Province (20IRTSTHN025). 


\section{Conflict of interest}

The author declare no conflict of interest.

\section{References}

Aiko, V., \& Mehta, A. (2015). Occurrence, detection and detoxification of mycotoxins. Journal of Biosciences, 40(5), 943-954. doi:10.1007/s12038-015-9569-6.

China, N. h. a. f. p. c. a. s. f. a. d. a. o. (2017). Determination of aflatoxin B1, B2, G1, G2 in grain:ultra-high performance liquid chromatography. In (Vol. LS/T 6128-2017(2017)).

Chu, F. S., Steinert, B., \& Gaur, P. (1985). Production and characterization of antibody against aflatoxin G1. Journal of Food Safety, 7(3), 161-170. doi: $10.1007 / \mathrm{bf0} 1949970$.

EC. (2006). (EC) No 1881/2006 setting maximum levels for certain contaminants in foodstuffs.

FDA. (2018). Adulteration with Aflatoxin-CPG Sec 555.400 Foods.

Gefen, T., Vaya, J., Khatib, S., Rapoport, I., Lupo, M., Barnea, E., ... Pitcovski, J. (2015). The effect of haptens on protein-carrier immunogenicity. Immunology, 144(1), 116-126. doi: 10.1111/imm.12356.

Guan, L. U., Zhang, Y. Z., Yang, T. T., You, J. M., HongMei, L. I., \& Sun, X. L. (2013). Synthesis and Characterization of the Hapten for Paraquat. Journal of Food ence \& Biotechnology, 32(5), 524-528. doi: 10.1016/S0926-860X(97)00036-7.

Iyer, R. S., \& Harris, T. M. (1993). Preparation of aflatoxin B1 8,9-epoxide using m-chloroperbenzoic acid. Chemical Research in Toxicology, 6(3), 313316. doi: 10.1021/tx00033a010.

Japan (2011). Management of food containing aflatoxin. No.0331-5.

Kim, S. H., Cha, S. H., Karyn, B., Park, S. W., \& Kang, H. G. (2011). Production of Group Specific Monoclonal Antibody to Aflatoxins and its Application to Enzyme-linked Immunosorbent Assay. Toxicological Research, 27(2), 125-131. doi: 10.5487/TR.2011.27.2.125.

Kortkhonjia, E., Brandman, R., Zhou, J. Z., Voelz, V. A., Chorny, I., Kabakoff, B., ... Swartz, T. E. (2013). Probing antibody internal dynamics with fluorescence anisotropy and molecular dynamics simulations. Microbiol Spectr, 5(2), 306-322. doi: 10.4161/mabs.23651.

Li, P., Zhou, Q., Wang, T., Zhou, H., \& Zhang, Q. (2015). Development of an Enzyme-Linked Immunosorbent Assay Method Specific for the Detection of G-Group Aflatoxins. Toxins, 8(1), 5. doi: 10.3390/toxins8010005.

Martin, C. N., \& Garner, R. C. (1977). Aflatoxin B-oxide generated by chemical or enzymic oxidation of aflatoxin B1 causes guanine substitution in nucleic acids. Nature, 267(5614), 863-865. doi: 10.1038/267863a0.
Rushing, B. R., \& Selim, M. I. (2019). Aflatoxin B1: A review on metabolism, toxicity, occurrence in food, occupational exposure, and detoxification methods. Food and Chemical Toxicology, 124, 81-100. doi: 10.1016/j.fct.2018.11.047.

Sevy, A. M., \& Meiler, J. (2014). Antibodies: ComputerAided Prediction of Structure and Design of Function. Microbiol Spectr, 2(6), 1-14. doi: 10.1128/ microbiolspec.AID-0024-2014.

Siahi Shadbad, M. R., Ansarin, M., Tahavori, A., Ghaderi, F., \& Nemati, M. (2012). Determination of aflatoxins in nuts of Tabriz confectionaries by ELISA and HPLC methods. Adv Pharm Bull, 2(1), 123-126. doi: 10.5681/apb.2012.018.

Wang, Y., Han, D., Wang, X., Lei, Z., Niu, L., \& Wang, Z. (2016). Preparation of Anti-Chromium Ion Monoclonal Antibodies and Analysis of Immunological Properties. Acta Agriculturae BorealiOccidentalis Sinica, 25(11), 1597-1602.

Wang, Y., Wang, S., Zhang, H., \& Wang, Z. (2014). Establishment of Hybridoma Cell Lines Secreting AntiCadmium Ion Monoclonal Antibody and Identification of Their Immunological Properties. Acta Agriculturae Boreali-Occidentalis Sinica, 23(11), 24-29.

Wu, F., Stacy, S. L., \& Kensler, T. W. (2013). Global Risk Assessment of Aflatoxins in Maize and Peanuts: Are Regulatory Standards Adequately Protective? Toxicological Sciences An Official Journal of the Society of Toxicology, 135(1), 251-259. doi: 10.1093/toxsci/kft132.

Xie, L., Chen, M., \& Ying, Y. (2016). Development of Methods for Determination of Aflatoxins. Crit Rev Food Sci Nutr, 56(16), 2642-2664. doi: 10.1080/10408398.2014.907234.

Xu, Z. L., Shen, Y. D., Beier, R. C., Yang, J. Y., Lei, H. T., Wang, H., \& Sun, Y. M. (2009). Application of computer-assisted molecular modeling for immunoassay of low molecular weight food contaminants: A review. Anal Chim Acta, 647(2), 125-136. doi: 10.1016/j.aca.2009.06.003.

Zhang, J., Li, P. W., Zhang, W., Zhang, Q., Ding, X., Chen, X., ... Zhang, X. (2009). Production and characterization of monoclonal antibodies against aflatoxin $\mathrm{g}(1)$. Hybridoma (Larchmt), 28(1), 67-70. doi: 10.1089/hyb.2008.0064.

Zhang, J. Y., Pei-Wu, L. I., Zhang, W., Ding, X. X., Liang, M. A., Wen-Hua, W. U., ... Tao, L. I. (2008). Synthesis of Aflatoxin G_1 Artificial Antigen. Food Science, 29(6), 194-197.

Zhou, Q., Zhang, W., Zhang, Q., Ding, X. X., \& Pei-Wu, L. I. (2010). Synthesis of aflatoxin G class artificial antigen. Chemical Reagents, 32(10), 859-872. 876. doi: 10.3788/AOS20103012.3552. 